EDITORIAL

\title{
A special issue on Silicon Photonics
}

\author{
Zhiping ZHOU (凶) \\ The State Key Laboratory of Advanced Optical Communication Systems and Networks, \\ School of Electronics Engineering and Computer Science, Peking University, Beijing 100871, China
}

(C) Higher Education Press and Springer-Verlag Berlin Heidelberg 2012

The tremendous demand on low cost optoelectronic systems that may be used for high-density data communications, real time sensing/detection and high-speed control/actuation has heated up the research and development in silicon photonics, which studies the principles and technologies of merging electronics and photonics into the silicon platform. To make a silicon photonic system reality, the compatibility in device size and integration processing between the photonics and electronics is essential. Over the past years, unprecedented advancement on individual silicon photonic devices, such as waveguide, beam splitter, light source, modulator, and detector, have been reported, yet the progress in size and processing issues are still under development.

To overcome the size incompatibility of the photonic devices and the electronic devices, the localization characters of the plasmonics and photonic crystal, and the high efficient smaller devices are brought into the play. On the other hand, the efforts in producing better coupling devices, better matched material systems are increased for tight integration of photonics and electronics. It is our intention to bring the research community's attention to these particular research areas. In this 'Special Issue on Silicon Photonics', 5 review articles and 11 research articles focusing on relevant subjects by internationally active groups in the field are specially presented.

More specifically, Prof. Ran of Peking University reviewed the transition from photonics to plasmonics from the point of view of the on-chip light source; Prof. Sun of Nankai University talked about the highly efficient silicon light emitting diodes through doping engineering; Prof. Xia of Huazhong University of Science and Technology presented his view on germanium quantum dots light-emitting devices; Dr. Li's group in the Institute of Physics, Chinese Academy of Sciences (CAS), presented their progresses on infrared two-dimensional silicon photonic crystal slab devices; and Dr. Cheng's group in the Institute of Semiconductor, CAS, summarized the recent development in photonic devices application of Ge-on-Si.

Among the research articles, Prof. Zhou's group and Prof. Huang's group showed their results on surface plasmon involved emissions. The following papers are aimed to improve the device performance and efficiency: Prof. Zhang's group and Dr. Yang's group targeted better and smaller waveguides; Prof. Zhou's group researched the binary blazed grating-based polarization-independent filter on silicon on insulator (SOI); Dr. Hou's group and Dr. Li's group reported their results on modulators by using quantum-confined Stark effect (QCSE) and Mach-Zehnder interferometer (MZI). From materials, integration, and system performance point of view, Prof. Su's group showed a compact all-optical differential-equation solver based on silicon microring resonator; Dr. Huang's group spelled their vision on design of unidirectional emission silicon/ III-V laser for on-chip interconnects; Prof. Xu's group and Dr. Huo's group researched on the annealing effect on the optical and electronic properties of non-stoichiometric amorphous silicon carbide films and the molecular beam epitaxy (MBE) growth of tensile-strained Ge quantum wells and quantum dots to obtain best conditions for on-chip hetero structured light sources.

Overall, articles in this special issue showcase the exciting achievements of silicon photonics, particularly in the interests of better devices and better integration. We hope the readers will find them inspiring and enjoyable.

Finally, we would like to express our appreciations to authors for devoting their precious time to write the intriguing articles and to reviewers for their helpful comments.

Received November 30, 2011

E-mail: zjzhou@pku.edu.cn 


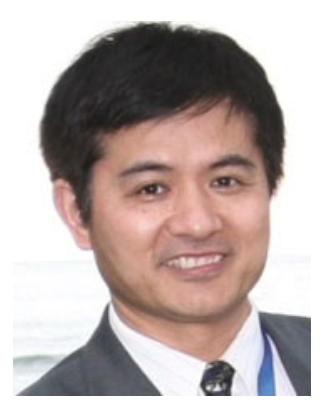

Zhiping (James) Zhou received his Ph.D. (EE) degree from Georgia Institute of Technology (GT), USA, in 1993. He was a Founder and the Vice President of Production at Hengnan Transistor Factory in China (1971-1978) and a guest scientist of NIST in USA (1987-1989). He is a Fellow of SPIE and IET, a senior member of IEEE and OSA, and a life member of PSC.

From 1993 to 2005, he was with the Microelectronics Research Center at GT, where he engaged research and development in the areas of semiconductor devices and sensors; photonic devices and sensors; ultra-fast optical communications; integrated optoelectronics; nanotechnology; and vector rigorous diffraction analysis. From 2005 to 2008, he was with Huazhong University of Science and Technology as a "Changjiang" Professor appointed by the Ministry of Education of China and since 2008, he is a "Changjiang" Professor at Peking University, Beijing, China, focusing on silicon photonics and microsystems research. He is also an Adjunct Professor at School of Electrical and Computer Engineering of GT, USA. He has over 240 technical papers and presentations, and holds 9 patents. 